\title{
ANATOMIA DA MADEIRA NA SUBTRIBO BACCHARINAE LESS. TENDÊNCIAS GERAIS DE ORDEM TAXONÔMICA E ECOLÓGICA ${ }^{1}$
}

\author{
JOSÉNEWTONCARDOSO MARCHIORI ${ }^{2}$ ANABELA SILVEIRADE OLIVEIRA-DEBLE $^{3}$
}

\section{RESUMO}

A semelhança anatômica da madeira, observada em cinco espécies de Baccharis (B. dracunculifolia, $B$. longoattenuata, B. milleflora, B. patens, B. tridentata), três de Heterothalamus (H. alienus, H. psiadioides, H. rupestris) e em Heterothalamulopsis wagenitzii, torna mais fácil o reconhecimento da subtribo Baccharinae, como um todo, do que os distintos gêneros. Esta semelhança pode ser atribuída tanto ao elevado grau evolutivo do xilema, como a uma especialização acelerada, de valor adaptativo à xeromorfia.

Palavras-chave: Taxonomia de madeiras, Anatomia ecológica, Asteraceae, Astereae, Baccharinae, Baccharis, Heterothalamus, Heterothamulopsis.

\section{SUMMARY}

[Wood anatomy in the subtribe Baccharinae Less.: Taxonomical and ecological trends]. The anatomical similarity, observed in five woody species of Baccharis (B. dracunculifolia, B. longoattenuata, B. milleflora, B. patens, B. tridentata), in the three species of Heterothalamus (H. alienus, H. psiadioides, $H$. rupestris) and in the wood of Heterothalamulopsis wagenitzii, makes easier to recognize a species as belonging to subtribe Baccharinae as a whole, than to a distinct genus. This similarity may be attributed to the high specialized xylem structure as much as to an accelerated specialization, due to xeromorphy.

Keywords: Wood taxonomy, Ecological anatomy, Asteraceae, Astereae, Baccharinae, Baccharis, Heterothalamus, Heterothalamulopsis.

\section{INTRODUÇÃO}

A abrangência do gênero Baccharis e sua circunscrição na taxonomia da subtribo Baccharinae variam consideravelmente entre os autores. Barroso (1976) e Barroso \& Bueno (2002), por exemplo, consideraram a existência de quatro gêneros para as Baccharinae brasileiras: Baccharidastrum, Baccharidiopsis, Baccharis e Heterothalamus. Giuliano (2000, 2001), em seu estudo sobre as espécies argentinas, reconheceu três gêneros: Baccharis, Baccharidastrum e Heterothalamus. Deble et al. (2004), acrescentaram um novo gênero para o Brasil: Heterothalamulopsis. Em publicação mais recente, Müller (2006) reduziu todos os gêneros pertencentes à subtribo Baccharinae, com exceção de Archibaccharis, à sinonímia de Baccharis L., que passou a compreender a totalidade das espécies brasileiras da referida subtribo botânica.

Os estudos de anatomia descritiva publicados pelos autores sobre madeiras de Heterothalamulopsis, Heterothalamus e Baccharis (Oliveira, Marchiori \& Deble, 2005; Oliveira, Deble \& Marchiori, 2005; Oliveira \& Marchiori, 2005; Marchiori, \& Oliveira, 2007; Marchiori, Oliveira-Deble \& Denardi, 2007), fornecem elementos suficientes para a apreensão das possibilida-

1 Recebido em 10/4/2007 e aceito para publicação em 30/4/2007, o presente trabalho científico é resultado parcial de projeto contemplado com Bolsa de Produtividade em Pesquisa, CNPq-Brasil.

2 Engenheiro Florestal, Dr., bolsista de Produtividade em Pesquisa do CNPq-Brasil, Professor Titular do Departamento de Ciências Florestais, Universidade Federal de Santa Maria, CEP 97105-900, Santa Maria(RS). balduinia@mail.ufsm.br

3 Bióloga, MSc., bolsista CAPES, doutoranda do Programa de Pós-Graduação em Engenharia Florestal, Centro de Ciências Rurais, Universidade Federal de Santa Maria, CEP 97105-900, Santa Maria (RS). anabela.biol.@mail.ufsm.br 
des estruturais do xilema no conjunto das Baccharinae, permitindo a formulação de considerações gerais de ordem taxonômica e ecológica.

\section{REVISÃO DE LITERATURA}

O emprego da estrutura interna das plantas como auxílio para a classificação vegetal é idéia muito antiga e tem sido utilizada com freqüência (Record, 1934). Em exsicatas de herbário desprovidas de órgãos reprodutivos, por exemplo, a análise da estrutura da madeira mostra-se de grande utilidade para a identificação, permitindo, não raro, a determinação de espécimes estéreis até família ou gênero. Na identificação de restos paleobotânicos, tais estudos são ainda imprescindíveis e autores, como Metcalfe \& Chalk (1972), postulam a utilização de métodos anatômicos em pesquisa taxonômica.

De acordo com Dadswell (1939), a anatomia de madeiras presta grande auxílio na classificação de certos gêneros botanicamente incertos, no estudo do arranjo interno de famílias e na determinação de prováveis afinidades entre famílias botânicas. Ocorre que grupos taxonômi$\cos$, como ordens e famílias botânicas, nem sempre apresentam um conjunto constante de caracteres estruturais (Chowdhury, 1959). Como exemplo, Chalk (1944) lembra que nas ordens Tiliales, Sterculiales e Malvales é mais fácil o reconhecimento de uma madeira como pertencente ao grupo como um todo, do que atribuí-la a uma determinada família, apesar de serem distintas algumas subfamílias destes táxones.

Comparada à taxonomia botânica, a anatomia da madeira baseia-se em menor número de caracteres e de espécies, motivo pelo qual suas informações tornam-se mais apropriadas como complemento das classificações naturais, aumentando o número de caracteres disponíveis para comparação. O anatomista de madeiras, segundo Chalk (1944), tende a comparar suas conclusões com aquelas da Botânica Sistemática.

A anatomia da madeira mostra-se especialmente valiosa no caso da classificação interna de famílias com grande variabilidade estrutural. A nível genérico, o xilema secundário é, usualmente, mais homogêneo, pois as pequenas diferenças morfológicas que distinguem espécies afins nem sempre se refletem na anatomia. Nesse contexto, torna-se essencial distinguir os caracteres que são relativamente constantes dos que são passíveis de variação, sob diferentes condições de crescimento. Sob esse ponto, Rendle (1944) observa que é necessário uma considerável experiência para se reconhecer os caracteres de real valor diagnóstico em uma madeira e estimar seu valor como típico de uma família, gênero ou espécie.

Algumas características, como frequiência de poros, comprimento de elementos vasculares e fração de parênquima axial, podem ser marcadamente influenciados pelo meio ambiente (Metcalfe \& Chalk, 1972). O diâmetro de vasos, mesmo variando segundo a posição na árvore e condições de crescimento, é freqüentemente reconhecido como importante para a identificação. Quanto aos raios, o caráter de maior utilidade é a sua largura, tanto em dimensão linear como em número de células; a altura também pode ser valiosa, sobretudo se os raios forem muito altos ou muito baixos. Raios exclusivamente unisseriados são de grande utilidade, podendo auxiliar na distinção de gêneros e espécies, principalmente se a característica for de ocorrência esporádica no grupo em questão (Metcalfe \& Chalk, 1972).

Com relação à anatomia ecológica, cabe lembrar, de início, que em todos os grupos de plantas vasculares encontram-se evidências da influência dos fatores mesológicos na evolução do xilema: ocorre que a estrutura da madeira resulta de um complexo mecanismo de interação, incluindo potencial genético e fatores do meio, que regula a atividade cambial e a morfogênese do xilema. Nesse processo, os principais fatores envolvidos resultam da adaptação ao grau de disponibilidade de água e taxa transpiratória, à oscilação sazonal desta disponibilidade, e a requisitos de reforço mecânico 
(Carlquist, 1975), podendo intensificar ou mesmo reverter tendências filogenéticas estabelecidas para determinadas espécies (Tsoumis, 1968; Graaf \& Baas, 1974).

De acordo com Carlquist (1966, 1969), existe uma estreita correlação entre xeromorfia e certas características anatômicas da madeira, como: elementos vasculares curtos e estreitos, poros em agrupamentos numerosos, raios baixos e presença de elementos imperfurados curtos. A correlação entre anatomia do xilema e hábito de crescimento foi abordado, entre outros, por Ayensu \& Stern (1964) e Walsh (1975). Segundo Carlquist (1975), elementos vasculares longos, especialmente os imperfurados, podem ser vantajosos para o suporte mecânico de espécies herbáceas, devido à produção limitada de xilema nestas plantas. Elementos vasculares curtos e de grandes diâmetros, ao contrário, são muito peculiares em lianas (Ayensu et al., 1964; Carlquist, 1975; Vliet, 1979). Poros de tamanhos variados, por sua vez, são mais freqüentes em lianas e xerófitas (Carlquist, 1985; Baas \& Schweingruber, 1985).

\section{ESPÉCIES ESTUDADAS}

Os dados anatômicos apresentados nas Tabelas 1 e 2, bem como as referências sobre a estrutura anatômica em espécies de Baccharis, Heterothalamus e Heterothalamulopsis, foram colhidas de publicações dos próprios autores, relacionadas na Bibliografia.

Com cerca de 400 espécies de ervas perenes, subarbustos, arbustos e pequenas árvores, o gênero Baccharis L. distribui-se desde o sul dos Estados Unidos até a Terra do Fogo (Giuliano, 2000). Das 146 espécies nativas no Brasil (Oliveira et al., 2006), foram selecionadas para estudo: Baccharis dracunculifolia, B. patens e B. tridentata, publicadas por Marchiori \& Oliveira (2007); e Baccharis longoattenuata e $B$. milleflora, descritas anatomicamente por Marchiori, Oliveira-Deble e Denardi (2007).

Arbusto de 2-3 m de altura, Baccharis dracunculifolia DC. distribui-se desde a Bolívia e Paraguai até o sul do Brasil e Uruguai. É uma das espécies pioneiras antrópicas mais fre- qüentes em capoeiras e margem de estradas e suas folhas, de 1-2,5 cm de comprimento por 3$4 \mathrm{~mm}$ de largura, são papiráceas e densamente pontuadas de glândulas (Barroso, 1976; Giuliano, 2000; Müller, 2006).

Restrito ao Uruguai e Rio Grande do Sul, Baccharis patens Baker é arbusto de 0,5-1,5 $\mathrm{m}$ de altura, com folhas de $1-2 \mathrm{~cm}$ de comprimento por 1-4 $\mathrm{mm}$ de largura, densamente tomentosas no dorso (Barroso, 1976).

Arbusto xilopodífero de $0,5-1 \mathrm{~m}$ de altura, Baccharis tridentata Vahl apresenta ampla distribuição no sul do Brasil, Paraguai, Uruguai e centro-norte da Argentina, tendo folhas de 1,5-5 $\mathrm{cm}$ de comprimento por $0,5-3 \mathrm{~cm}$ de largura (Barroso, 1976; Giuliano, 2000).

Descrita recentemente (Oliveira \& Deble, 2006), Baccharis longoattenuata é uma das espécies de maior porte no gênero, alcançando, alguns indivíduos, cerca de $15 \mathrm{~m}$ de altura.

Arbusto de ramos alados e folhas bracteiformes, Baccharis milleflora (Less.) DC. habita sobretudo em solos úmidos de áreas campestres, no sudeste do Brasil e Uruguai (Müller, 2006).

O gênero Heterothalamus Less., reduzido, por Müller (2006), à sinonímia de Baccharis L., compreende apenas três espécies de distribuição geográfica pampeana (Waechter, 2002). Destas, Heterothalamus alienus (Sprengel) O. Kuntze é a de distribuição geográfica mais ampla, ocorrendo de Santa Catarina ao Uruguai, bem como em área disjunta no centro da Argentina. Arbusto de 1-5 m de altura, apresenta folhas lineares, íntegras, de 7-30 mm de comprimento por $0,7 \mathrm{~mm}$ de largura.

De menor porte $(0,5-2 \mathrm{~m})$, Heterothalamus psiadioides Less. distingue-se facilmente pelas folhas oblongas, serreadas na metade superior; sua distribuição geográfica inclui os Aparados da Serra Geral, em Santa Catarina e Rio Grande do Sul, e parte da Serra do Sudeste, sendo elemento raro no Uruguai.

Descrito há pouco tempo (Deble, Oliveira \& Marchiori, 2003), Heterothalamus rupestris é subarbusto lenhoso (0,5-1,5 m) de ocorrência restrita ao Rio Grande do Sul, que habita 
afloramentos rochosos úmidos, em pontos esparsos da Serra do Sudeste. Suas folhas, de $10-30 \mathrm{~mm}$ de comprimento por 1-1,5 mm de largura, apresentam 1-6 dentes na metade superior.

\section{ANÁLISE ANATÔMICA}

Os caracteres anatômicos resumidos na Tabela 1 demonstram que as cinco espécies de Baccharis, as três de Heterothalamus e Heterothalamulopsis wagenitzii são muito semelhantes entre si com relação à estrutura anatômica da madeira, apresentando as seguintes características em comum: porosidade difusa, vasos de diâmetro extremamente pequeno a muito pequeno $(<50 \mu \mathrm{m})$, elementos vasculares muito curtos $(<300 \mu \mathrm{m})$, placas de perfuração simples, pontoações intervasculares alternas, parênquima paratraqueal, raios heterogêneos, raios multisseriados com 2-4 células de largura e fibras libriformes extremamente curtas $(<750$ $\mu \mathrm{m})$, não septadas, providas de pontoações simples muito pequenas. Além de comuns a todas as espécies em estudo, tais características são, também, de larga ocorrência nas Asteraceae ou Compositae, segundo Metcalfe \& Chalk (1972) e Record \& Hess (1949).

Como visto na Tabela 2, todas as madeiras estudadas apresentam vasos com diâmetro mé- dio inferior a $50 \mu \mathrm{m}$, e não apenas Heterothalamus wagenitzii $(23 \mu \mathrm{m})$, que é um subarbusto, mas até mesmo Baccharis longoattenuata (32 $\mu \mathrm{m})$ que, de porte médio, é uma das espécies de maior tamanho, tanto no gênero Baccharis como na tribo Astereae. Apesar do porte arbóreo geralmente indicar mesomorfia nas Compositae (Carlquist, 1966), a estrutura anatômica é claramente xeromórfica em Baccharis longoattenuata, à semelhança do verificado nas demais espécies estudadas. De acordo com Carlquist (1960), o diâmetro reduzido dos vasos nas Astereae pode ser atribuído tanto à posição filética mais elevada da tribo, dentro das Compositae, como à especialização acelerada, decorrente da xeromorfia.

A presença de vasos extremamente pequenos é outro caráter anatômico marcante na tribo Astereae (Carlquist, 1966), contrastando neste aspecto com as Cichorieae (Carlquist, 1960), mas não com as Helenieae (Carlquist, 1959). Mesmo assim, alguns vasos com diâmetro superior a $100 \mu \mathrm{m}$ podem ser encontrados em certas espécies de Baccharis (B. cassiniaefolia, B. concava, B. neglecta, B. thesioides), e dos gêneros Haplopappus, Lepidospartum, Olearia e Psiadia (Carlquist, 1960).

TABELA 1. Caracteres observados nas madeiras de Baccharis dracunculifolia (B. d.), B. longoattenuata (B. 1.), B. milleflora (B. m.), B. patens (B. p.), B. tridentata (B. t.), Heterothalamus alienus (H. a.), H. psiadioides (H. p.), H. rupestris (H. r.) e Heterothalamulopsis wagenitzii (H. w.).

\begin{tabular}{|c|c|c|c|c|c|c|c|c|c|}
\hline Caráter anatômico & B. d. & B. $l$. & B. $m$. & B. p. & B.t. & H. a. & H. p. & H. $r$. & H. w. \\
\hline 1. $\varnothing \operatorname{Vasos}<50 \mu \mathrm{m}$ & $\mathrm{x}$ & $\mathrm{x}$ & $\mathrm{x}$ & $\mathrm{x}$ & $\mathrm{x}$ & $\mathrm{x}$ & $\mathrm{x}$ & $\mathrm{x}$ & $\mathrm{x}$ \\
\hline 2. Vasos em padrão dendrítico & $\mathrm{x}$ & $\mathrm{x}$ & & $\mathrm{x}$ & & $\mathrm{x}$ & $\mathrm{x}$ & $\mathrm{x}$ & $\mathrm{x}$ \\
\hline 3. Vasos em padrão radial & & & $\mathrm{x}$ & & $\mathrm{x}$ & & & & \\
\hline 4.Comprimento elementos vasculares $<300 \mu \mathrm{m}$ & $\mathrm{x}$ & $\mathrm{x}$ & $\mathrm{x}$ & $\mathrm{x}$ & $\mathrm{x}$ & $\mathrm{x}$ & $\mathrm{x}$ & $\mathrm{x}$ & $\mathrm{x}$ \\
\hline 5. Espessamentos espiralados & $\mathrm{x}$ & $\mathrm{x}$ & & $\mathrm{x}$ & $\mathrm{x}$ & $\mathrm{x}$ & $\mathrm{x}$ & & $\mathrm{x}$ \\
\hline 6. Placas de perfuração simples & $\mathrm{x}$ & $\mathrm{x}$ & $\mathrm{x}$ & $\mathrm{x}$ & $\mathrm{x}$ & $\mathrm{x}$ & $\mathrm{x}$ & $\mathrm{x}$ & $\mathrm{x}$ \\
\hline 7. Pontoações intervasculares alternas & $\mathrm{x}$ & $\mathrm{x}$ & $\mathrm{x}$ & $\mathrm{x}$ & $\mathrm{x}$ & $\mathrm{x}$ & $\mathrm{x}$ & $\mathrm{x}$ & $\mathrm{x}$ \\
\hline 8. Parênquima paratraqueal & $\mathrm{x}$ & $\mathrm{x}$ & $\mathrm{x}$ & $\mathrm{x}$ & $\mathrm{x}$ & $\mathrm{x}$ & $\mathrm{x}$ & $\mathrm{x}$ & $\mathrm{x}$ \\
\hline 9. Raios heterogêneos & $\mathrm{x}$ & $\mathrm{x}$ & $\mathrm{x}$ & $\mathrm{x}$ & $\mathrm{x}$ & $\mathrm{x}$ & $\mathrm{x}$ & $\mathrm{x}$ & $\mathrm{x}$ \\
\hline 10. Raios multisseriados com 2-4 células & $\mathrm{x}$ & $\mathrm{x}$ & $\mathrm{x}$ & $\mathrm{x}$ & $\mathrm{x}$ & $\mathrm{x}$ & $\mathrm{x}$ & $\mathrm{x}$ & $\mathrm{x}$ \\
\hline 11. Fibras libriformes & $\mathrm{x}$ & $\mathrm{x}$ & $\mathrm{x}$ & $\mathrm{x}$ & $\mathrm{x}$ & $\mathrm{x}$ & $\mathrm{x}$ & $\mathrm{x}$ & $\mathrm{x}$ \\
\hline 12. Fibras não septadas & $\mathrm{x}$ & $\mathrm{x}$ & $\mathrm{x}$ & $\mathrm{x}$ & $\mathrm{x}$ & $\mathrm{x}$ & $\mathrm{x}$ & $\mathrm{x}$ & $\mathrm{x}$ \\
\hline 13. Comprimento médio de fibras $<750 \mu \mathrm{m}$ & $\mathrm{x}$ & $\mathrm{x}$ & $\mathrm{x}$ & $\mathrm{x}$ & $\mathrm{x}$ & $\mathrm{x}$ & $\mathrm{x}$ & $\mathrm{x}$ & $\mathrm{x}$ \\
\hline 14. Cristais ausentes & & $\mathrm{x}$ & $\mathrm{x}$ & & $\mathrm{x}$ & $\mathrm{x}$ & $\mathrm{x}$ & $\mathrm{x}$ & $\mathrm{x}$ \\
\hline 15. Cristais presentes & $\mathrm{x}$ & & & $\mathrm{x}$ & & & & & \\
\hline
\end{tabular}

$\varnothing=$ diâmetro; $\mu \mathrm{m}=$ micrômetros. 
TABELA 2. Dados quantitativos médios, para as madeiras de Baccharis dracunculifolia (B. d.), B. longoattenuata (B. 1.), B. milleflora (B. m.), B. patens (B. p.), B. tridentata (B. t.), Heterothalamus alienus (H. a.), H. psiadioides (H. p.), H. rupestris (H. r.) e Heterothalamulopsis wagenitzii (H. w.).

\begin{tabular}{|c|c|c|c|c|c|c|c|c|c|}
\hline Caráter anatômico & B. d. & B. $l$. & B. $m$. & B. $p$. & B. $t$. & H. $a$. & H. p. & H. $r$. & H. w. \\
\hline 1. $\varnothing \operatorname{vasos}(\mu \mathrm{m})$ & 28 & 32 & 27 & 25 & 36 & 35 & 35 & 31 & 23 \\
\hline 2. Comprimento elementos vasculares $(\mu \mathrm{m})$ & 202 & 230 & 204 & 143 & 198 & 154 & 209 & 162 & 177 \\
\hline 3. Comprimento de fibras $(\mu \mathrm{m})$ & 646 & 704 & 519 & 521 & 572 & 572 & 580 & 463 & 451 \\
\hline
\end{tabular}

$\varnothing=$ diâmetro; $\mu \mathrm{m}=$ micrômetros.

Para as nove espécies examinadas, além do diâmetro reduzido, chama atenção a elevada freqüência de vasos (poros $/ \mathrm{mm}^{2}$ ) e seu agrupamento em numerosos múltiplos, aspectos anatômicos de valor sabidamente adaptativo em espécies xeromórficas, pois proporcionam maior capilaridade por unidade de volume da madeira. Com exceção de Baccharis milleflora e $B$. tridentata, cujos múltiplos seguem um padrão radial, o arranjo de vasos nas demais espécies é nitidamente dendrítico.

Ainda com relação aos vasos, cabe salientar que só não foram observados espessamentos espiralados em Baccharis milleflora e Heterothalamus rupestris. O que mais chama atenção, neste caso, é que a ausência do caráter manifesta-se, justamente, em duas espécies de morfologia indiscutivelmente xeromórfica: a primeira delas, por ser arbusto de caules alados e folhas bracteiformes; a segunda, por seu menor porte, comparado a Heterothalamus alienus, e por habitar afloramentos rochosos, como indicado no próprio nome. Ao aumentarem a superfície interna dos vasos, os espessamentos espiralados favorecem a capilaridade, constituindo estratégia anatômico-ecológica de reconhecido valor adaptativo à xeromorfia. $\mathrm{A}$ presença do caráter, em algumas espécies de Baccharis, e sua completa ausência, em outras, não constitui novidade, tendo sido observada por Carlquist (1960). Aparente paradoxo, a ausência de espessamentos espiralados nos vasos de Baccharis milleflora e Heterothalamus rupestris aponta para a necessidade de novos estudos anatômicos, incluindo outras espécies da seção Caulopterae DC. (ou subgênero Molina (Persoon) Heering), com vistas ao esclarecimento de eventuais significados filéticos e/ou evolutivos para o conjunto dos Baccharis, e seus diferentes grupos infragenéricos (seções ou subgêneros).

A ocorrência de elementos vasculares muito curtos $(<300 \mu \mathrm{m})$ nas nove espécies em estudo, traz a vantagem de proporcionar maior número de placas de perfuração por unidade de volume de madeira, favorecendo, por conseguinte, a capilaridade no xilema secundário: trata-se, portanto, de mais um caráter anatômico de significado ecológico-adaptativo. A presença de elementos vasculares curtos, por outro lado, também atesta um alto grau evolutivo ao xilema de Baccharis, Heterothalamus e Heterothalamulopsis.

Em todas as espécies estudadas ocorrem fibras extremamente curtas $(<750 \mu \mathrm{m})$, como indicado na Tabela 2. Para todas as espécies, como esperado, este comprimento excede ao dos elementos vasculares. As fibras libriformes de pontoações simples diminutas, observadas nas nove espécies de Baccharinae estudadas, além de claro indício de especialização do xilema (Metcalfe \& Chalk, 1972), constituem caráter generalizado na tribo Astereae, segundo Carlquist (1960).

Ao contrário da maioria das Compositae (Record \& Hess, 1949), nas espécies em estudo não foram observados septos em fibras; caráter raro na tribo Astereae, a presença de fibras septadas foi referida apenas para Olearia cunninghamii, por Carlquist (1960). 
Com relação ao raios, o material estudado segue o padrão referido para as Astereae: raios heterogêneos com células procumbentes e quadradas, ou células quadradas e eretas, ou ambos. Com relação aos multisseriados, em todas as espécies verificaram-se raios de 2-4 células de largura. Caráter raro em Compositae (Metcalfe \& Chalk, 1972), a presença de cristais foi anotada apenas para Baccharis dracunculifolia e $B$. patens, no material em estudo; a apresença do caráter, todavia, não constitui novidade para as Astereae, sendo referido, por Carlquist (1960), para Baccharis angustifolia, B. pilularis e Aster spinosus, entre outras espécies da tribo.

Como dito anteriormente, a anatomia da madeira demonstra grande semelhança estrutural nas cinco espécies de Baccharis aqui consideradas (B. dracunculifolia, B. longoattenuata, B. milleflora, B. patens, B. tridentata), bem como nas três de Heterothalamus ( $H$. alienus, $H$. psiadioides, $H$. rupestris) e em Heterothalamulopsis wagenitzii, fato que pode ser atribuído tanto ao elevado grau evolutivo do xilema, traço marcante na tribo Astereae e família Asteraceae, como à especialização acelerada, de valor adaptativo à xeromorfia. Os caracteres diferenciais entre espécies, como presença de espessamentos espiralados e cristais, não contestam a homogeneidade estrutural das Baccharinae e tribo Astereae, por não serem circuncritos a determinado gênero. A anatomia, em suma, demonstra ser mais fácil o reconhecimento de uma madeira como pertencente à subtribo Baccharinae, como um todo, do que a um determinado gênero em especial.

A redução dos gêneros Heterothalamus e Heterothalamulopsis à sinonímia de Baccharis L., proposta de Müller (2006), não pode ser contestada à luz da anatomia da madeira; mesmo assim, cabe lembrar que o instrumental anatômico, baseado em número relativamente pequeno de caracteres, serve mais como complemento para as classificações naturais, do que, propriamente, para opiniões definitivas em Taxonomia Botânica.

\section{BIBLIOGRAFIA}

Ayensu, E. S., Stern, W. L. Systematic anatomy and ontogeny of the stem in Passifloraceae. Contr. U.S. Nat. Herb., v. 34, n. 3, p. 45-73, 1964.

Baas, P., Schweingruber, F. H. Ecological trends in the wood anatomy of trees, shrubs and climbers from Europe. Iawa Bulletin, v. 8, n. 3, p. 245-274, 1985.

Barroso, G. M. Compositae - Subtribo Baccharidinae, Estudo das espécies ocorrentes no Brasil. Rodriguésia, n. 40, p. 1-273, 1976.

Barroso, G. M., Bueno, O. L. Compostas. 5. Subtribo Baccharidinae. In: Flora Ilustrada Catarinense. Itajaí: Herbário Barbosa Rodrigues, 2002. p. 7651064.

Carlquist, S. Wood anatomy of Helenieae (Compositae). Tropical Woods, v. 111, p. 19-39, 1959.

Carlquist, S. Wood anatomy of Cichorieae (Compositae). Tropical Woods, v. 112, p. 61-91, 1960.

Carlquist, S. Wood anatomy of Compositae: a summary with comments on factors controlling wood evolution. Aliso, v. 6, n. 2, p. 25-44, 1966.

Carlquist, S. Wood anatomy of Goodeniaceae and the problem of insular woodiness. Ann. Missouri Bot. Garden, v. 56, p. 358-390, 1969.

Carlquist, S. Ecological strategies of xylem evolution. Berkeley: University of California Press, 1975.259p.

Carlquist, S. Observations on wood functional histology of vines and lianas; vessel dimorphism, tracheids, vasicentric tracheids, narrow vessels and parenchyma. Aliso, v. 11, p. 139-157, 1985.

Chalk, L. On the taxonomic value of anatomical structure of the vegetative organs of the Dicolyledons. 2. The taxonomic value of wood anatomy. Proc. Linn. Soc. London, v. 155, n. 3, p. 214-218, 1944.

Chowdhury, K. A. Limitations of anatomical aid to taxonomy of Angiosperms. In: International Botanical Congress, 9, Montreal, 1959. Recent Advances in Botany, v. 1, p. 150-155.

Dadswell, H. E. The role of wood anatomy in forest botany. J. Counc. Sci. Indust. Res. Aust., n. 12, p. 237-242, 1939.

Deble, L. P., Oliveira, A. S. de, Marchiori, J. N. C. Heterothalamus rupestris, espécie nova de Asteraceae do Rio Grande do Sul. Ciência Florestal, Santa Maria, v. 13, n. 2, p. 1-5, 2003. 
Deble, L. P., Oliveira, A. S. de, Marchiori, J. N. C. Heterothalamulopsis, gênero novo da subtribo Baccharinae Lessing (Astereae - Asteraceae). Ciência Florestal, Santa Maria, v. 14, n. 1, p. 1-7, 2004.

Giuliano, D. Subtribo Baccharidinae. In: Hunziker, A. T. Flora Fanerogámica Argentina. Cordoba, 2000. v. $66,73 \mathrm{p}$.

Giuliano, D. Clasificación infragenérica de las especies argentinas de Baccharis (Asteraceae Astereae). Darwiniana, n. 39, p. 131-154, 2001.

Graaf, N. A., Baas, P. van der. Wood anatomical variation in relation to latitude and altitude. Blumea, v. 22, p. 101-121, 1974.

Marchiori, J. N. C., Oliveira, A. S. de. Anatomia da madeira de três espécies brasileiras de Baccharis L. (Asteraceae-Astereae). Balduinia, Santa Maria, n. 10, p. 1-10, 2007.

Marchiori, J. N. C., Oliveira-Deble, A. S. de, Denardi, L. Anatomia da madeira de duas espécies brasileiras de Baccharis L. (Asteraceae-Astereae). Balduinia, Santa Maria, n. 11, p.1-8, 2007.

Metcalfe, C. R., Chalk, L. Anatomy of the Dicotyledons. Oxford: Clarendon Press, 1972.1500 p.

Müller, J. Systematics of Baccharis (CompositaeAstereae) in Bolivia, including an overview of the genus. Ann Arbor: The American Society of Plants Taxonomists, 2006. 341 p. (Systematic Botany Monographs, v. 76).

Oliveira, A. S. de, Deble, L. P., Marchiori, J. N. C. Anatomia da madeira de duas espécies do gênero Heterothalamus Lessing (Asteraceae Astereae), nativas no Rio Grande do Sul. Ciência Florestal, Santa Maria, v. 15, n. 1, p. 9-19, 2005.
Oliveira, A. S. de., Marchiori, J. N. C., Deble, L. P. Anatomia do lenho de Heterothalamus wagenitzii (F. Hellw.) Deble, Oliveira \& Marchiori (Asteraceae - Astereae). Balduinia, Santa Maria, n. 2, p. 13-18, 2005.

Oliveira, A. S. de, Marchiori, J. N. C. Anatomia do lenho de Heterothalamus psiadioides Lessing (Asteraceae-Astereae). Balduinia, Santa Maria, n. 4, p. 20-24, 2005.

Oliveira, A. S. de, Deble, L. P. Duas novas espécies sul-brasileiras de Baccharis L. (Asteraceae Astereae). Balduinia, Santa Maria, n. 9, p. 4-9, 2006.

Oliveira, A. S. de, Deble, L. P., Marchiori, J. N. C. Checklist do gênero Baccharis L. para o Brasil (Asteraceae - Astereae). Balduinia, Santa Maria, n. 9, p. 17-27, 2006.

Record, S. J. Role of the Wood Anatomy in Taxonomy. Tropical Woods, v. 37, p. 1-9, 1934.

Record, S. J., Hess, R. W. Timbers of the New World. New Haven: Yale University Press, 1949.640 p.

Rendle, B. J. On the taxonomic value of the anatomical structure of the vegetative organs of Dicotyledons. 3. The role of anatomy in the practical identification of commercial timbers. Proc. Linn. Soc. London, v. 155, n. 3, p. 218-221, 1944.

Tsoumis, G. Wood as raw material. Oxford: Pergamon Press, 1968.276 p.

Vliet, G. J. C. M. van. Wood anatomy of the Combretaceae. Blumea, v. 25, p. 141-223, 1979.

Waechter, J. L. Padrões geográficos na flora atual do Rio Grande do Sul. Ciência \& Ambiente, n. 24, p. 93-108, 2002.

Walsh, M. A. Xylem anatomy of Hibiscus (Malvaceae) in relation to habit. Botanical Gazette, v. 136, p. 30-40, 1975. 